\section{A) Check for updates}

Cite this: Food Funct., 2019, 10, 7204

\title{
Exploring the effects of Gastrodia elata Blume on the treatment of cerebral ischemia-reperfusion injury using UPLC-Q/TOF-MS-based plasma metabolomics $\uparrow$
}

\author{
Dongxu Wang, (D) a Qi Wang, ${ }^{\mathrm{b}}$ Renhao Chen, ${ }^{\mathrm{a}}$ Shinlin Yang, ${ }^{\mathrm{b}}$ Zhifeng Li*a and \\ Yulin Feng*b
}

\begin{abstract}
Gastrodia elata Blume (Orchidaceae, GEB) is a medicinal plant that has been widely used in the treatment of cerebrovascular disease. This study explored the protective effects of GEB against cerebral ischemiareperfusion using Information-Dependent Acquisition (IDA)-mediated UPLC-Q/TOF-MS-based plasma metabolomics. Cerebral ischemia-reperfusion (IR) injury was induced in male Wistar rats using the Zea Longa method. Biochemical and histological assays were performed to evaluate the therapeutic effects of GEB on IR rats. We found that the neurobehavioral scores and infarction areas of GEB and nimodipine treated groups were dramatically lower than those of the IR groups. Hematoxylin and Eosin (HE) staining and TdT-mediated dUTP Nick-End Labeling (TUNEL) showed that GEB significantly improved neuronal injury and prevented neuronal apoptosis. Biochemical analysis revealed that GEB prevented cerebral ischemia-reperfusion injury by regulating inflammation and oxidative injury. Through ultra-high-performance liquid chromatography-quadrupole time-of-flight mass spectrometry-metabolomics-based approaches, 43 plasma metabolites related to GEB treatment were detected, 6 of which significantly differed $(p<0.05)$ between the model and GEB groups. The levels of L-histidine, sphinganine, thymine, spermidine and deoxycytidine in the IR group were significantly higher than those in the sham group, but decreased following GEB treatment. Arachidonic acid levels were lower in the IR group, but dramatically increased in response to GEB. Pharmacodynamics and metabolomics confirmed that the mechanism of GEB in the treatment of cerebral ischemia was not only related to the reduction of inflammation, oxidation, neurotoxicity, and apoptosis, but also mediated through arachidonic acid metabolism, histidine metabolism, pyrimidine metabolism, arginine and proline metabolism, sphingolipid metabolism, and glycerophospholipid metabolism in vivo.
\end{abstract}

Accepted 20th September 2019

DOI: 10.1039/c9fo01729a

rsc.li/food-function tissue-type plasminogen activator (t-PA) significantly decreases stroke related disability and is the front-line treatment for cerebrovascular disease. ${ }^{4}$ Conventional intravenous thrombolysis, however, fails to reverse the ischemic death of nerve cells, leading to injury and apoptosis $;^{5}$ t-PA also leads to side effects including a reduced level of consciousness and reduced language ability, and also has strict restrictions on the physique of patients and therapeutic time window. ${ }^{4}$ These limitations highlight the need for new and more effective therapeutic interventions.

In recent years, Gastrodia elata Blume (GEB) has emerged as effective in the treatment of convulsions, vertigo, headaches, hemiplegia, limb numbness and rheumatoid arthritis. ${ }^{6}$ In addition to the inherent advantages of traditional Chinese medicine (including reduced toxicity, fewer side effects, stable curative effects and syndrome differentiation) GEB is a functional food that has been recorded in the ancient book "Materia a Jiangxi University of Traditional Chinese Medicine, Nanchang 330002, China. E-mail: lizhifeng1976@hotmail.com

${ }^{b}$ State Key Laboratory of Innovative Drug and Efficient Energy-Saving Pharmaceutical Equipment, Nanchang 330006, China. E-mail: fengyulin2003@126.com

$\dagger$ Electronic supplementary information (ESI) available. See DOI: 10.1039/ c9fo01729a 
Medica Yan Yi". GEB has been incorporated into functional foods including porridge, wine, preserved fruit, and beverages, with the aim of enhancing immunity, slowing oxidation, reducing blood lipid levels and blood pressure, improving fatigue, and promoting relaxation. GEB has also been suggested as a promising therapeutic for cerebrovascular disease. ${ }^{7}$

GEB has favorable therapeutic effects on ischemic disease through its ability to dilate cerebral vessels, improve the microcirculation, inhibit the release of NO and NOS, protect the blood-brain barrier, ${ }^{8}$ protect brain cells from oxidative stress and inflammatory injury ${ }^{9,10}$ and inhibit apoptosis and autophagy. ${ }^{11}$ Cerebral ischemia-reperfusion injury is the main pathological process of ischemic cerebrovascular disease ${ }^{12}$ as it promotes free radical injury, calcium overload, amino acid metabolism, nitric oxide production, inflammation, and apoptosis. ${ }^{12-16}$ Cerebral disease due to middle cerebral artery occlusion accounts for $\sim 70 \%$ of the total clinical cases. ${ }^{17}$ In this study, the MCAO model was used to simulate clinical cerebral ischemia to explore the therapeutic effects of GEB.

Through the analysis of metabolites in vivo, genotypes and phenotypes can be linked and biomarkers can be identified, which enhance our understanding of disease processes at the molecular level. ${ }^{18}$ In terms of TCM, metabolomics permits the evaluation of key processes that permit therapeutic efficacy. An Information-Dependent Acquisition (IDA)-mediated LC-MS/MS method is an advanced approach in the qualitative study of many target compounds and has been successfully used to support plasma pharmacokinetic screening programs. In this study, liquid phase mass spectrometry (LC-MS) was used to explore the therapeutic mechanisms of GEB on cerebral ischemia-reperfusion injury in rats. We aimed to identify potential disease biomarkers to provide new avenues for the prevention and treatment of ischemic stroke.

\section{Experimental approaches}

\subsection{Materials}

Medicinal materials were collected from the Heilongjiang Province and identified as dry roots and rhizomes or stems of GEB by Professor Guoyue Zhong of the Jiangxi University of Traditional Chinese Medicine. As a control, 2-chloro-L-phenylalanine (purity $>98 \%$ ) was purchased from Shanghai McLean Biochemical Technology Co., Ltd. Acetonitrile and methanol (HPLC grade) were purchased from Fisher Scientific (MA, USA). Formic acid (analysis grade) was purchased from China Chemical Reagent Co., Ltd. Nimodipine was purchased from the YABAO Pharmaceutical Group Co., Ltd. Triphenyl tetrazolium chloride (TTC) and Hematoxylin and Eosin (HE) staining kits were purchased from Beijing Solarbio Science \& Technology Co., Ltd. Interleukin-6 (IL-6), Interleukin-1 $\beta$ (IL-1 $\beta)$, Tumor Necrosis Factor $\alpha(\mathrm{TNF}-\alpha)$ and Elisa kits were purchased from NEOBIOSCIENCE technology (Shenzhen, China). Superoxide dismutase (SOD), glutathione peroxidase (GSH-Px) and malondialdehyde (MDA) kits were purchased from the Nanjing Jiancheng Bioengineering Institute (Nanjing,
China). TdT-mediated dUTP Nick-End Labeling (TUNEL) apoptosis assay kits and proteinase $\mathrm{K}$ were purchased from Beyotime Biotechnology (Shanghai, China). Other equipment included multi-functional enzyme labeling instruments (SpectraMax i3, Molecular Devices); a high-speed freezing centrifuge (HC-3018R, Anhui Zhongke Zhongjia Scientific instrument Co., Ltd); an automatic sample grinding instrument (J288/48, Shanghai Jingxin Technology); and rat brain coronal section models (Shenzhen Ruiwod). The sections were imaged on a LEICA DM 2500, Leica Microsystems Co., Ltd. A dehydrator (Zhejiang Jinhua Cody instrument and equipment Co., Ltd) and a liquid vacuum concentrated decoction machine (YZN50, Beijing Donghua original Medical Equipment Co., Ltd) were used for GEB preparation.

\subsection{Preparation of GEB extracts}

Roots and rhizomes of GEB (500 g) were washed, dried and ground into a crude powder. The samples were then decocted three times through $70 \%$ ethanol reflux $(5 \mathrm{~L})$ for 2 hours each time. Combined extracts were filtered at high temperature, concentrated under vacuum at $60{ }^{\circ} \mathrm{C}$, and freeze-dried. GEB extracts were dissolved in sodium carboxymethyl cellulose aqueous solution to prepare a $200 \mathrm{mg} \mathrm{kg}^{-1}$ suspension for rat administration.

\subsection{Study design}

2.3.1. Animals. Briefly, adult male Wistar rats $(260 \pm 20 \mathrm{~g})$ were obtained from Beijing (SCXK (京) 2016-0006). All animals were fed a standard diet and water. Experimental conditions were maintained thermostatically at $22 \pm 1{ }^{\circ} \mathrm{C}$ with $55 \pm 5 \%$ humidity and a 12-light/12-dark cycle. Animal studies were performed in compliance with the requirements for the Guide for the Care and Use of Laboratory Animals at the National Institutes of Health (NIH Publication No. 80-23; revised 1978). Experiments were recognized by the Animal Care and Use Committee of the Jiangxi University of Chinese Medicine.

2.3.2. Groups and drug administration. Rats were randomly divided into 4 groups ${ }^{19-22}$ ( $n=10$ each) including: (1) sham-operated controls (sham); (2) IR (0.9\% NaCl); (3) GEB (200 $\left.\mathrm{mg} \mathrm{kg}^{-1}\right)$; and (4) nimodipine groups $\left(15 \mathrm{mg} \mathrm{kg}^{-1}\right)$ as a comparative positive control. All rats were intragastrically administered the indicated treatment regimens once a day for 15 days. After 2 hours of final administration, the rats were anesthetized through the intraperitoneal injection of $10 \%$ chloral hydrate (350 $\mathrm{mg} \mathrm{kg}^{-1}$ ).

2.3.3. IR injury. The model of middle cerebral artery occlusion (middle cerebral artery occlusion, MCAO) is generally regarded as the standard animal model of focal cerebral ischemia. $^{23,24}$ Rat models of focal cerebral ischemia produced through the Longa method ${ }^{25}$ are recognized as the gold standard. The Zea Longa method ${ }^{19}$ was used to establish IR in rats. Rats were anesthetized through the intraabdominal injection of chloral hydrate $\left(350 \mathrm{mg} \mathrm{kg}^{-1}\right)$ and fixed in the supine position. The right external carotid artery (ECA), the internal carotid artery (ICA) and the common carotid artery (CCA) were fully exposed by an incision in the middle of the neck. The 
proximal end of the CCA and root of the ECA were separated and ligated. A small incision was introduced at the area between the ligations of the two arteries. A thread (length: $50 \mathrm{~mm}$, diameter: $0.32 \pm 0.02 \mathrm{~mm}$ ) was slowly inserted into the internal carotid artery from the common carotid artery and tied. After two hours, the thread was pulled for $\sim 15 \mathrm{~mm}$ to establish blood reperfusion. All treatment methods were performed in the sham group, but the thread was not inserted into the internal carotid artery.

\subsection{Neurobehavioral observations and TTC staining}

The neurobehavioral scores of the rats were assigned from 0 to 4 points. $^{26}$ After 24 hours of reperfusion, no observable neurological dysfunction was marked as 0 points. Failure to extend the left forelimb upon lifting the tail was marked as 1 point. An inclination to crawl in the opposite direction was marked as 2 points. Autonomous turning to the opposite side as opposed to walking was marked as 3 points. Failure to spontaneously walk for an extended time period or a loss of consciousness was marked as 4 points. Rats were euthanized by decapitation after neurobehavioral assessments. Their brains were sectioned into six coronal slices of $2 \mathrm{~mm}$ thickness to determine the infarct volume with TTC staining. Positive and negative sides of the sections were incubated at $37^{\circ} \mathrm{C}$ for $8 \mathrm{~min}$ and fixed in $4 \%$ paraformaldehyde for 3-5 hours. The brain slices were stained and imaged to measure the total area and infarct size using image analysis software (Image-ProPlus 6.0).

\subsection{HE and TUNEL staining}

After 24 hours of reperfusion, blood from the abdominal aorta was collected and the aortas were immediately clamped. A perfusion needle was inserted into the left ventricle and the right auricle was cut open. The samples were perfused with saline for $10 \mathrm{~min}$ and $4 \%$ paraformaldehyde for $20 \mathrm{~min}$. The brain tissue between the root of the optic chiasma and the corpora quadrigemina was fixed in $4 \%$ paraformaldehyde. Fixed tissues were dehydrated and embedded to prepare continuous paraffin sections, which were subjected to HE and TUNEL staining and imaged using a fluorescence microscope.

\subsection{Biochemical evaluation}

All rats were decapitated after 24 hours of reperfusion. Fresh brain tissue was removed and washed with chilled saline solution. Excess saline was removed with filter paper and coronal sections around the bregma point were collected $(1 \mathrm{~mm})$. Coronal brains were weighed after sectioning. Brain tissues were added to precooled saline at a ratio of $1: 9$ and homogenized and centrifuged for $10 \mathrm{~min}$ (4000 rpm). The supernatant was collected and frozen at $-80{ }^{\circ} \mathrm{C}$. The activities of SOD, GSH-Px and MDA contents in the brain tissue were measured using commercial enzymatic assays. IL- $1 \beta$, IL-10 and TNF- $\alpha$ levels were determined by ELISA.

\subsection{Metabolomics analysis}

2.7.1. Sample pretreatment. Metabolomics was performed in rats from sham, IR, and GEB groups. Plasma supernatants were treated with methanol containing 2-chloro-L-phenylalanine $\left(10 \mu \mathrm{g} \mathrm{m} \mathrm{m}^{-1}\right)$ at a ratio of $4: 1$ (methanol:plasma supernatant, $\mathrm{V} / \mathrm{V}, 250 \mu \mathrm{l})$. The samples were centrifuged (12 $000 \mathrm{rpm}, 20 \mathrm{~min})$ at $4{ }^{\circ} \mathrm{C}$ and supernatants were filtered using $0.22 \mu \mathrm{m}$ microporous filter membranes. The samples were analyzed by LC-MS and $50 \mu \mathrm{l}$ of all the centrifuged supernatants were evenly mixed as the QC sample. ${ }^{27}$

2.7.2. LC-MS analysis. A Shimadzu UHPLC (ESI) system and an $\mathrm{AB}$ Sciex quadrupole time-of-flight mass spectrometer (TripleTOF® 5600) were used for LC-MS analysis. An ACQUITY UPLC C18 column $(100 \mathrm{~mm} \times 2.1 \mathrm{~mm}, 1.7 \mu \mathrm{m}$, water $)$ was used at a flow rate of $0.3 \mathrm{~mL} \mathrm{~min}^{-1}$, and an injection volume of $2 \mu \mathrm{L}$. Using $0.1 \%$ formic acid (A) and acetonitrile (B) with water as the mobile phase, the gradient of mobile phase $\mathrm{B}$ was as follows: $2-30 \%$ (0-3 $\mathrm{min}), 30-60 \%$ (3-5 $\mathrm{min}), 60-80 \%$ (5-15 $\mathrm{min}), 80-100 \%$ (15-16 $\mathrm{min}), 100 \%$ from 16 to $19 \mathrm{~min}$, 100-2\% (19-20 $\mathrm{min}$ ) and $2 \%$ held from 20 to $25 \mathrm{~min}$. TOF-MS and TOF-MS/MS were performed simultaneously. The TOF-MS mass ranged from 50 to 1250 . When the collision was $40 \mathrm{eV}$, the most intense 8 ions from each TOF-MS scan were selected for TOF-MS/MS.

To ensure data quality, previously described approaches were adopted. ${ }^{9,26}$ TOF-MS was calibrated every 5 samples to maintain data accuracy and stability. ${ }^{28}$ Simultaneous LC-MS analysis was performed for QC $(n=6)$ and plasma samples. The relative standard deviations (RSDs) of the retention times and typical peak intensities (including internal standards) in the QC samples were used to evaluate data quality.

2.7.3. Data analysis. Data were analyzed as previously described. ${ }^{26,29}$ The raw data of the LC-MS were firstly transformed into the " $\mathrm{m} / \mathrm{z}$ " data file format using Markerview 1.2 software, and the abundance of aligned features was normalized through internal standards (2-chloro-L-phenylalanine). The detection frequencies (DFs) and RSDs of each group were used for data screening. The features were subjected to statistical analysis only when the DFs of any group reached $100 \%$ and the RSD was less than $30 \%$. Missing values were replaced by the half minimum of abundant features. PCA (Principal Component Analysis) and OPLS-DA (orthogonal projectionpotential structure discriminant analysis) were used to ensure the quality of data and the model's reliability, a default 7-fold cross-validation and testing with 200 random permutations were performed using SIMCA-P software to avoid the overfitting of the OPLS-DA models, features with VIP scores $>1$ in the OPLS-DA model, with $p$-values $<0.05$, were selected and their candidate metabolites were identified with the Human Metabolome Database (HMDB). Associated metabolic pathways were established through integration of the Small Molecule Pathway Database (SMPDB)/KEGG and MetaboAnalyst $4.0 .^{30-32}$ The metabolites which have high impact on the metabolic pathway and cause significant changes in the plasma of IR rats are identified as potential biomarkers after reference compound confirmation. ${ }^{29,33}$ A Student's $t$-test was used for all data analysis. Differences were considered significant when $P<0.05$. 


\section{Results}

\subsection{Efficiency of GEB treatment}

3.1.1. Neurobehavioral scores and cerebral infarction evaluation. The neurobehavioral scores of the IR group were significantly higher $(p<0.001)$ than those of the sham group. The cerebral infarction area was consistent with these scores (Fig. 1). TTC staining (Fig. 2) and scoring scales proved the effectiveness of the cerebral ischemic injury model. Both the GEB and nimodipine treated groups showed significantly lower scores (both $p<0.05)$ and infarction areas $(p<0.05$; $p<0.001$ ) compared to the IR groups. GEB has a favorable therapeutic effect on nerve injury and cerebral ischemiareperfusion injury, although the effects are less pronounced than those in the nimodipine group.

3.1.2. HE and TUNEL staining. HE (Fig. 3) and TUNEL staining (Fig. 4) showed that GEB treatment significantly improved the loose edema of the parietal cortex. GEB and nimodipine also reduced nuclear pyknosis, nerve cell atrophy, peripheral demyelination, neuronal degeneration and microglial hyperplasia in rats induced by IR injury. Apoptosis was induced when cerebral cells were broken under the catalysis of terminal deoxynucleotidyl transferase. The 3-OH of the broken genomic DNA combined with FITC-labeled DUPT and exhibited fluorescence. TUNEL staining showed the presence of a large number of apoptotic cells in the parietal cortex of IR groups, the percentage of which significantly declined in the presence of GEB.

3.1.3. Biochemical evaluation. During ischemia and cerebral reperfusion, oxidative stress is enhanced and ROS production increases, leading to tissue injury. To explore the effects of GEB on antioxidant systems, we evaluated the activities of MDA, SOD, and GSH-Px. The brain MDA level in the IR group significantly increased to $11.08 \pm 0.7179 \mathrm{nmol} \mathrm{mL}^{-1}$, whilst GSH-Px and SOD levels significantly decreased to $49.48 \pm 5.006 \mu \mathrm{mol} \mathrm{mL} \mathrm{m}^{-1}$ and $73.81 \pm 2.792 \mu \mathrm{mol} \mathrm{mL} \mathrm{m}^{-1}$ compared to the sham group. GEB-treatment significantly reduced the MDA levels to $9.15 \pm 0.2915 \mathrm{nmol} \mathrm{mL}^{-1}$, whilst the activity

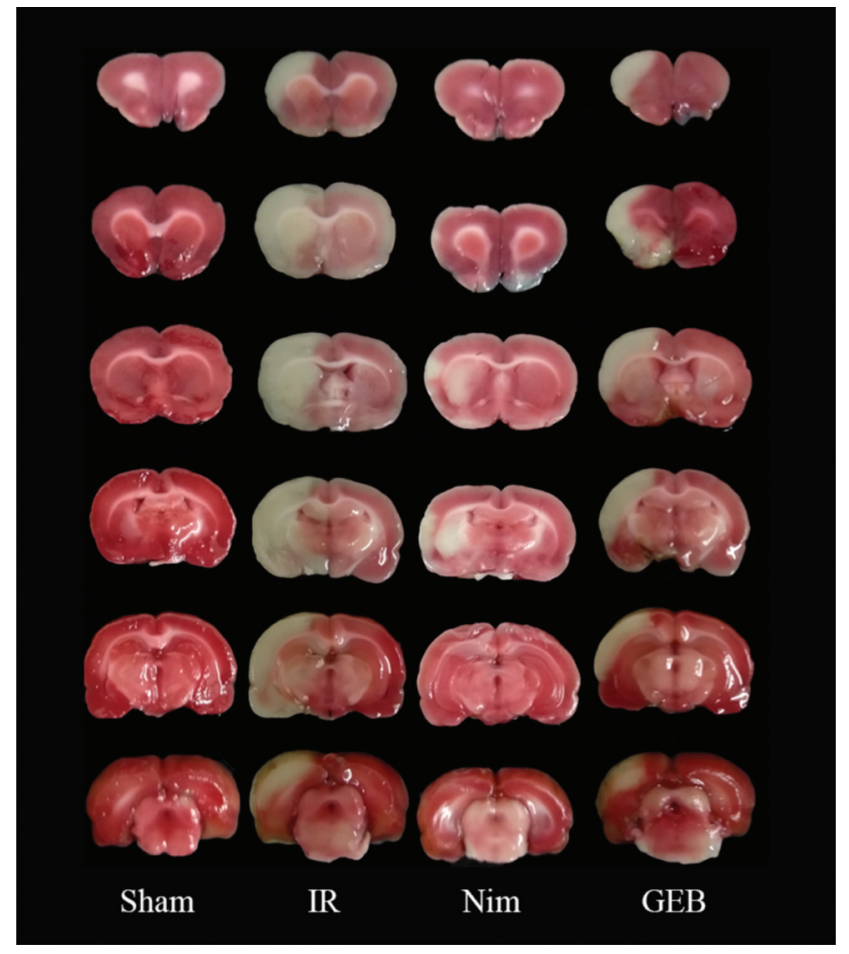

Fig. 2 Effects of GEB on the morphology of cerebral tissue observed by TTC staining in rats with cerebral ischemia reperfusion injuries.

of SOD and GSH-Px increased to $93.31 \pm 3.248 \mathrm{U} \mathrm{mL}^{-1}$ and $51.21 \pm 1.89 \mathrm{U} \mathrm{mL}^{-1}$ (Table 1). These indicators supported the role of GEB in recovering cerebral damage and reducing necrosis in vivo post-IR.

In IR injury rats, IL-10 expression dramatically decreased $(p<0.001)$ whilst IL-1 $\beta$ and TNF- $\alpha$ levels significantly increased $(p<0.001)$ compared to the sham group. Compared to the IR injury group, a significant improvement in IL-10 $(p<0.01)$ was observed in the GEB group. However, GEB pretreatment effectively blocked the increase in IL-1 $\beta$ and TNF- $\alpha$ compared to the IR group $(p<0.01 ; p<0.05)$ (Table 2$)$. These results suggested
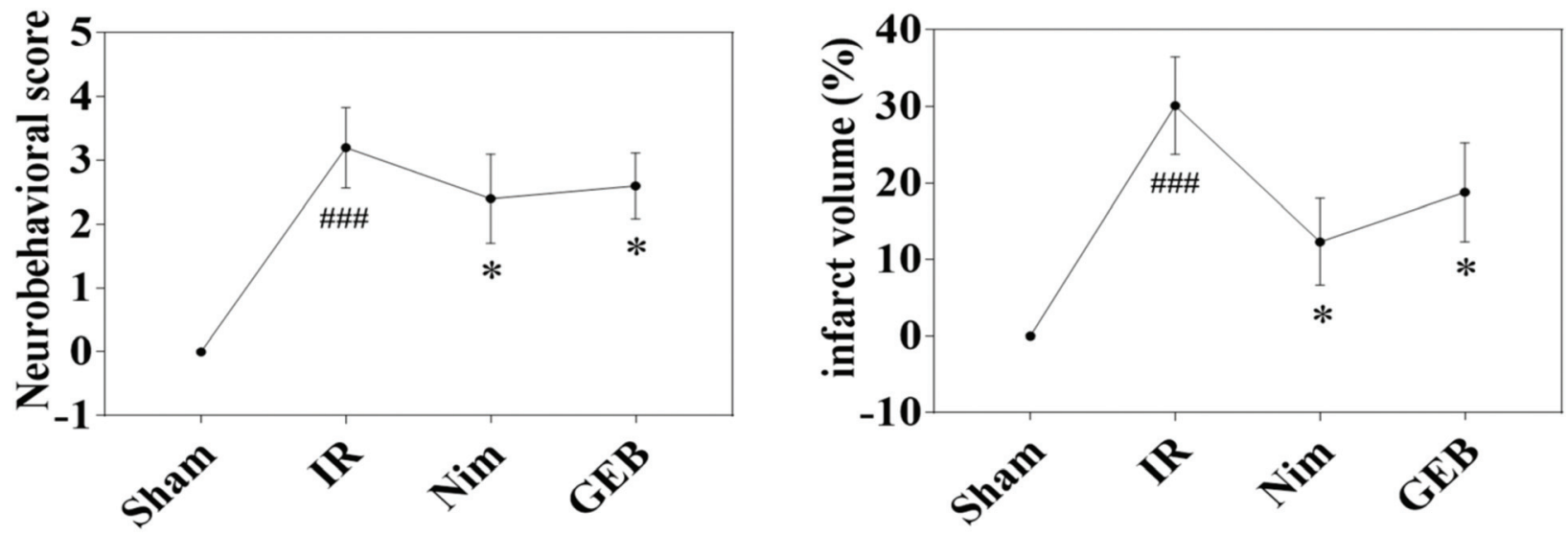

Fig. 1 Protective effect of GEB on neurological defects and infarct area in the ischemia-reperfusion rats $(\bar{x} \pm s) .{ }^{*} p<0.05$ vs. IR, \#\#\#p<0.001 vs. sham. 


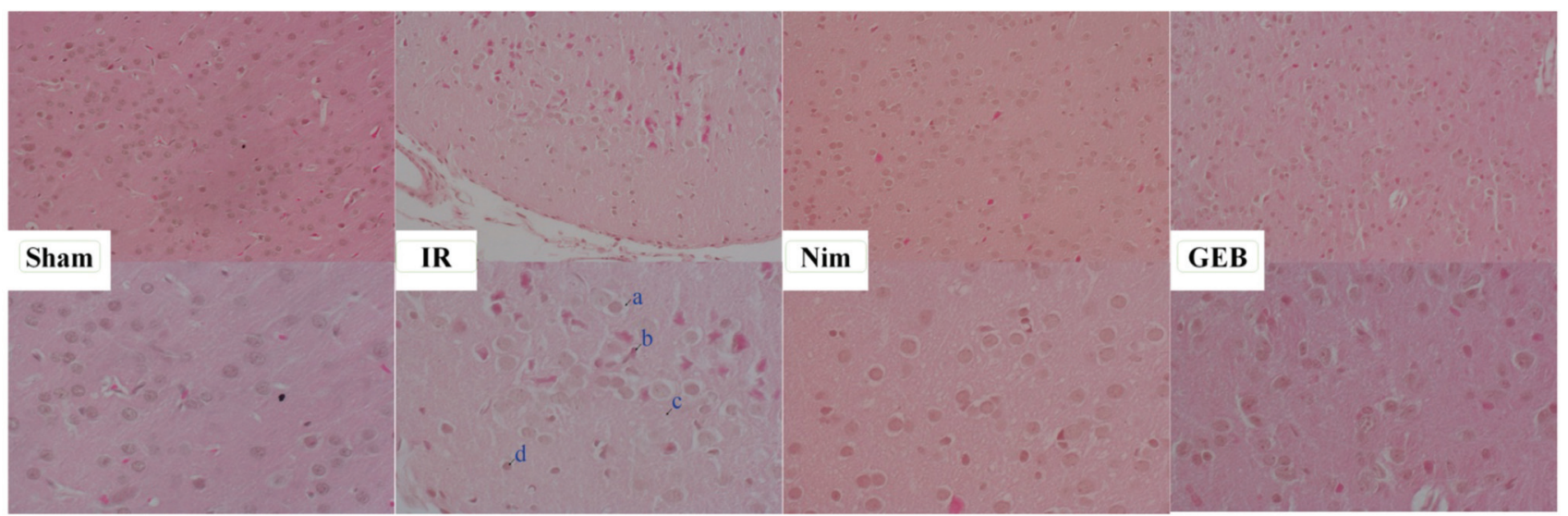

Fig. 3 HE staining of the parietal cortex. GEB and nimodipine reduced peripheral demyelination (a), nuclear pyknosis, nerve cell atrophy and neuronal degeneration (b); the loose edema of the parietal cortex (c); and microglial hyperplasia (d) in rats induced by IR injury.
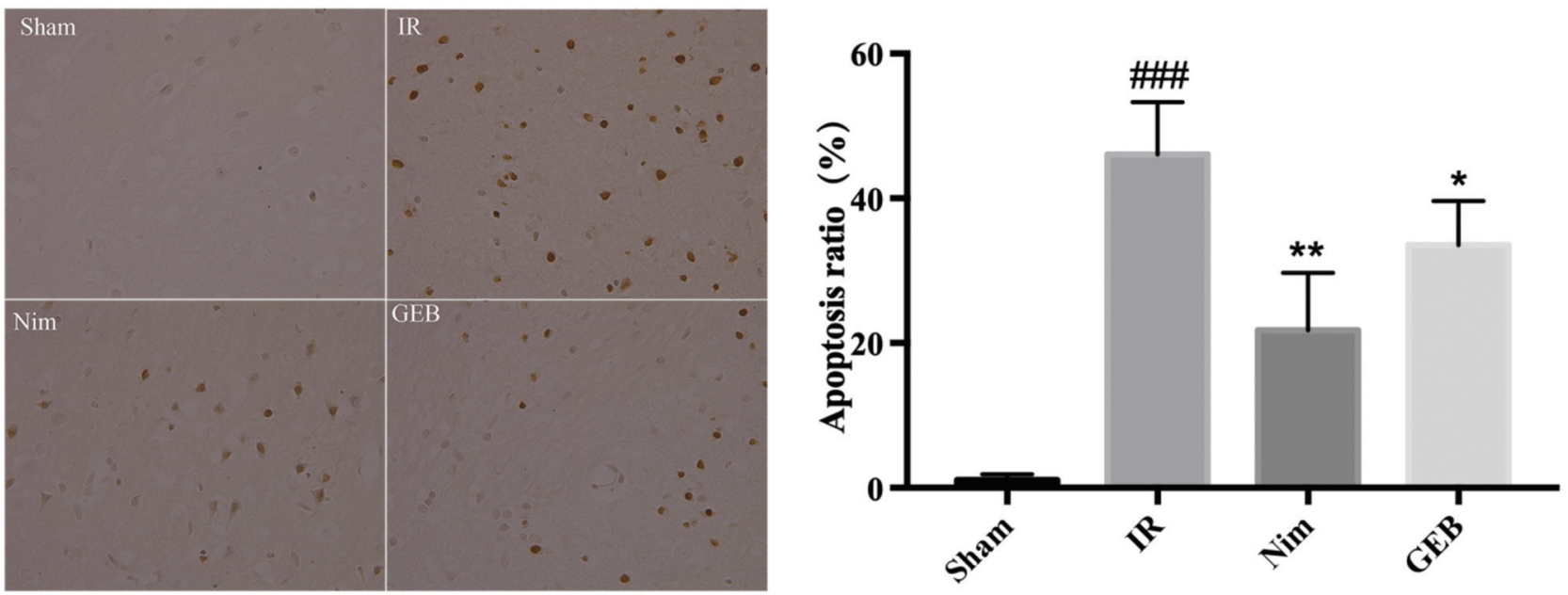

Fig. 4 The result of TUNEL staining and the apoptotic ratio. ${ }^{*} p<0.05$ vs. IR, ${ }^{*} p<0.01$ vs. IR, \#\#\#p $<0.001$ vs. sham.

Table 1 Effects of GEB on SOD, GSH-Px and MDA contents in brain tissues of rats with cerebral IR injuries $(\bar{x} \pm s)$

\begin{tabular}{llll}
\hline Groups & $\begin{array}{l}\text { SOD (U per } \\
\text { mg prot) }\end{array}$ & $\begin{array}{l}\text { GSH-Px } \\
\text { (U per mg prot) }\end{array}$ & $\begin{array}{l}\text { MDA (nmol per } \\
\text { mg prot) }\end{array}$ \\
\hline Sham & $117.5 \pm 4.877^{*}$ & $79.43 \pm 1.599^{*}$ & $7.605 \pm 0.5745^{*}$ \\
IR & $73.81 \pm 2.792$ & $49.48 \pm 5.006$ & $11.08 \pm 0.7179$ \\
Nimodipine & $92.77 \pm 2.837^{*}$ & $67.19 \pm 2.614^{*}$ & $8.933 \pm 0.6169$ \\
GEB & $93.31 \pm 3.248^{*}$ & $51.21 \pm 1.89$ & $9.15 \pm 0.2915^{*}$
\end{tabular}

${ }^{*} p<0.05, v s$. IR; mg prot means mg (protein).

that GEB plays a significant role in the anti-inflammatory mechanisms of cerebral ischemia and regulates the expression of inflammatory factors in ischemic cerebral tissue.

\subsection{Metabolic responses of rat to GEB treatment}

3.2.1. Verification of the metabolomics methods. The 10 typical ion peaks in the QC samples showed a high degree of overlap in the peak intensity and retention times in positive
Table 2 Effects of GEB on IL-1 $\beta$, IL-10 and TNF- $\alpha$ contents in brain tissues of rats with cerebral IR injuries $(\bar{x} \pm s)$

\begin{tabular}{llll}
\hline Groups & $\mathrm{IL}-10\left(\mathrm{ng} \mathrm{L}^{-1}\right)$ & $\mathrm{IL}-1 \beta\left(\mathrm{ng} \mathrm{L}{ }^{-1}\right)$ & $\mathrm{TNF}-\alpha\left(\mathrm{ng} \mathrm{L}^{-1}\right)$ \\
\hline Sham & $595 \pm 48^{*}$ & $269.5 \pm 21.22^{*}$ & $1101 \pm 61.38^{*}$ \\
IR & $332.2 \pm 31.98$ & $443.4 \pm 20.17$ & $1934 \pm 183$ \\
Nimodipine & $422.4 \pm 32.9$ & $366.9 \pm 30.73^{*}$ & $1378 \pm 98.36^{*}$ \\
GEB & $458.8 \pm 26.54^{*}$ & $373.7 \pm 21.26^{*}$ & $1443 \pm 80.5^{*}$
\end{tabular}

${ }^{*} p<0.05, v s$. IR.

and negative ion modes (Fig. S1 $\uparrow$ ), combined with the good clustering of QC samples in the PCA plot (Fig. 5); the data quality was found to meet the requirements for statistical analysis and confirmed the reproducibility of the methods. ${ }^{29}$

The signal responses of plasma metabolites in the ESI+ and ESI- modes were combined to analyze their distribution. The results showed significant differences in the metabolites between the two modes. In Fig. 6, PCA plots and OPLS-DA plots showed that samples of the sham, model and GEB 

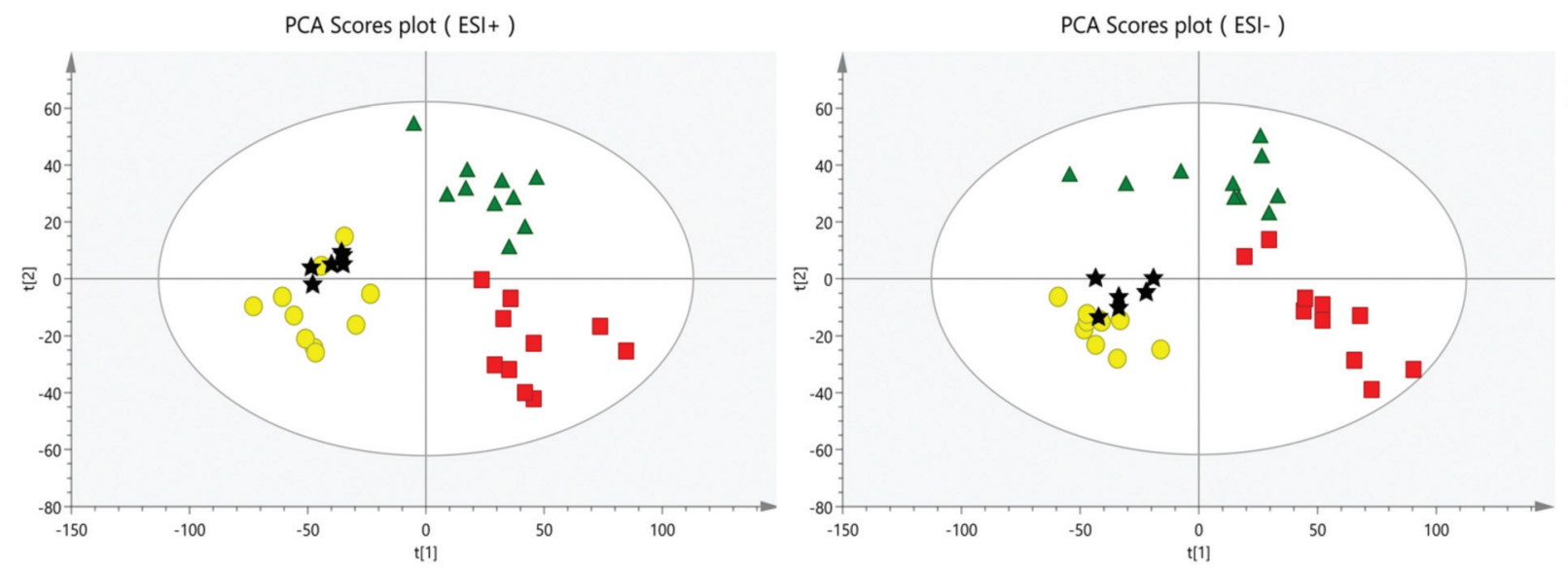

Fig. 5 PCA score plot for quality control (QC) validation.

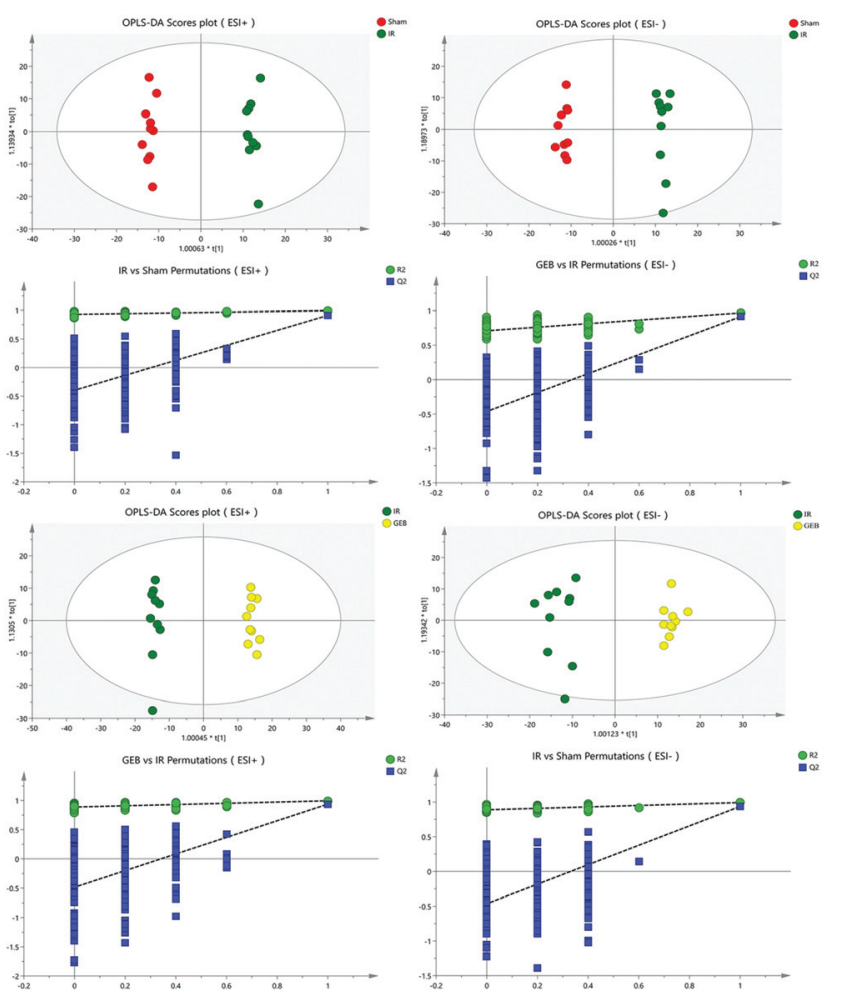

Fig. 6 OPLS-DA and permutation score plots of sham, IR and GEB groups.

groups had good clustering and permutation plots showed no overfitting of the model. The quality of the OPLS-DA is often assessed using R2Y and Q2Y. In this study, the R2Y and Q2Y were 0.993 and 0.912 in positive mode and 0.995 and 0.937 in the negative mode, respectively. This indicated that the quality of the OPLS-DA model was reliable.

3.2.2. Identification of potential biomarkers. Features with VIP scores $>1$ in the OPLS-DA model, with $p$-values $<0.05$, were selected and the precursor ions and MS/MS fragments obtained using UPLC-QTOF-MS/MS with high resolution were matched with information of metabolites provided by the online database HMDB; the metabolites showing an error between the extraction mass value and experimental mass value of less than $10 \mathrm{ppm}$ and matching these conditions were identified as candidate biomarkers. Finally, a total of 43 metabolites (Table 3) were identified as candidate biomarkers. To identify the metabolites that differed among the control, model and GEB groups, thermograms of the 43 candidate biomarkers (Fig. 7) were measured and compared. Color changes on the maps indicated the overall changes in metabolites. For instance, thymine, spermidine, L-carnitine, ureidosuccinic acid, sphingosine, and deoxycytidine were of higher levels in IR compared to the sham/GEB groups, and LysoPC (20:1(11Z)), arachidonic acid, and LysoPE (18:0/0:0) were of lower abundance in GEB compared to IR groups, suggesting a correlation following GEB treatment. Altered metabolites $(p<0.05)$ between the model and GEB groups were further evaluated for their response to GEB treatment. Associated metabolic pathways were established through integration of the Small Molecule Pathway Database (SMPDB)/KEGG and MetaboAnalyst 4.0. Six metabolites including thymine, spermidine, L-histidine, deoxycytidine, sphinganine, and arachidonic acid were selected (Fig. 8). These metabolites, after acquisition, injection and identification of reference compounds, represent potential biomarkers to verify the treatment efficacy of GEB.

\subsection{Metabolic pathway enrichment analysis}

Through the enrichment of 43 metabolites, a metabolism pathway with a higher score was constructed, including 6 potential biomarkers with significant changes in the plasma of IR rats. The main metabolism pathways involved in these metabolites were identified: namely pyrimidine metabolism, arginine and proline metabolism, glycerophospholipid metabolism, sphingolipid metabolism, histidine metabolism and arachidonic acid metabolism. GEB can treat cerebral ischemiareperfusion injury by affecting the above-mentioned pathways. The pathway impact is shown in Fig. $\mathrm{S} 2 \dagger$ and details of the metabolic pathways are shown in Fig. 9. 
Table 3 Potential biomarkers of cerebral ischemic injury post-treatment with GEB

\begin{tabular}{|c|c|c|c|c|c|c|c|}
\hline$M / Z$ & RT & Formula & Name & Adduct & HMDB & IR vs. sham & GEB vs. IR \\
\hline 86.096 & 1.45 & $\mathrm{C}_{5} \mathrm{H}_{11} \mathrm{~N}$ & Piperidine & $+\mathrm{H}$ & HMDB0034301 & $\downarrow$ & $\downarrow^{* * *}$ \\
\hline 127.0488 & 1.75 & $\mathrm{C}_{5} \mathrm{H}_{6} \mathrm{~N}_{2} \mathrm{O}_{2}$ & Thymine & $+\mathrm{H}$ & HMDB0000262 & $\uparrow^{* *}$ & $\downarrow^{*}$ \\
\hline 146.1645 & 0.8 & $\mathrm{C}_{7} \mathrm{H}_{19} \mathrm{~N}_{3}$ & Spermidine & $+\mathrm{H}$ & HMDB0001257 & $\uparrow^{*}$ & $\downarrow^{* * *}$ \\
\hline 150.0571 & 1.06 & $\mathrm{C}_{5} \mathrm{H}_{11} \mathrm{NO}_{2} \mathrm{~S}$ & L-Methionine & $+\mathrm{H}$ & HMDB0000696 & $\uparrow$ & $\downarrow^{* * *}$ \\
\hline 162.1139 & 2.08 & $\mathrm{C}_{6} \mathrm{H}_{11} \mathrm{NO}_{2}$ & L-Carnitine & $+\mathrm{H}$ & HMDB0000062 & $\uparrow^{* * * *}$ & $\downarrow^{* * *}$ \\
\hline 176.0648 & 1.44 & $\mathrm{C}_{5} \mathrm{H}_{8} \mathrm{~N}_{2} \mathrm{O}_{5}$ & Ureidosuccinic acid & $+\mathrm{NH}_{4}-\mathrm{H}_{2} \mathrm{O}$ & HMDB0000828 & $\uparrow^{*}$ & $\downarrow^{* * *}$ \\
\hline 178.0595 & 0.84 & $\mathrm{C}_{6} \mathrm{H}_{9} \mathrm{~N}_{3} \mathrm{O}_{2}$ & L-Histidine & $+\mathrm{Na}$ & HMDB0000177 & $\uparrow^{*}$ & $\downarrow^{* * *}$ \\
\hline 188.0696 & 2.01 & $\mathrm{C}_{11} \mathrm{H}_{11} \mathrm{NO}_{3}$ & Indolelactic acid & $+\mathrm{H}-\mathrm{H}_{2} \mathrm{O}$ & HMDB0000671 & $\uparrow^{* * *}$ & $\downarrow^{*}$ \\
\hline 204.1234 & 0.94 & $\mathrm{C}_{9} \mathrm{H}_{14} \mathrm{O}_{4}$ & L-Acetylcarnitine & $+\mathrm{H}$ & HMDB0000201 & $\uparrow^{* * *}$ & $\downarrow$ \\
\hline 226.1789 & 15.88 & $\mathrm{C}_{13} \mathrm{H}_{25} \mathrm{NO}_{3}$ & $N$-Undecanoylglycine & $+\mathrm{H}-\mathrm{H}_{2} \mathrm{O}$ & HMDB0013286 & $\uparrow^{* * *}$ & $\downarrow^{* *}$ \\
\hline 246.1724 & 3.59 & $\mathrm{C}_{12} \mathrm{H}_{25} \mathrm{NO}_{5}$ & Valerylcarnitine & $+\mathrm{H}$ & HMDB0013128 & $\uparrow^{* * *}$ & $\downarrow$ \\
\hline 249.0608 & 2.71 & $\mathrm{C}_{8} \mathrm{H}_{13} \mathrm{~N}_{2} \mathrm{O}_{5} \mathrm{P}$ & Pyridoxamine 5'-phosphate & $+\mathrm{H}$ & HMDB0001555 & $\uparrow$ & $\downarrow^{* *}$ \\
\hline 250.0795 & 1.21 & $\mathrm{C}_{9} \mathrm{H}_{13} \mathrm{~N}_{3} \mathrm{O}_{4}$ & Deoxycytidine & $+\mathrm{Na}$ & HMDB0000014 & $\uparrow^{* * *}$ & $\downarrow^{* * *}$ \\
\hline 279.0894 & 12.91 & $\mathrm{C}_{12} \mathrm{H}_{14} \mathrm{~N}_{4} \mathrm{O}_{2} \mathrm{~S}$ & Sulfamethazine & $+\mathrm{H}$ & HMDB0015522 & $\uparrow$ & $\downarrow^{* * *}$ \\
\hline 288.2529 & 9.03 & $\mathrm{C}_{16} \mathrm{H}_{33} \mathrm{NO}_{3}$ & Lauroyl diethanolamide & $+\mathrm{H}$ & HMDB0032358 & $\uparrow^{* * *}$ & $\downarrow^{* * *}$ \\
\hline 295.1275 & 3.3 & $\mathrm{C}_{14} \mathrm{H}_{18} \mathrm{~N}_{2} \mathrm{O}_{5}$ & Glutamylphenylalanine & $+\mathrm{H}$ & HMDB0029156 & $\uparrow^{* * *}$ & $\downarrow$ \\
\hline 296.0661 & 0.87 & $\mathrm{C}_{10} \mathrm{H}_{15} \mathrm{~N}_{3} \mathrm{O}_{5}$ & Glycerophosphocholine & $+\mathrm{K}$ & HMDB0000086 & $\downarrow^{*}$ & $\downarrow^{* *}$ \\
\hline 302.3054 & 8.95 & $\mathrm{C}_{18} \mathrm{H}_{39} \mathrm{NO}_{2}$ & Sphinganine & $+\mathrm{H}$ & HMDB0000269 & $\uparrow^{* *}$ & $\downarrow^{* * *}$ \\
\hline 437.1952 & 9.42 & $\mathrm{C}_{26} \mathrm{H}_{30} \mathrm{O}_{7}$ & Obacunone & $+\mathrm{H}-\mathrm{H}_{2} \mathrm{O}$ & HMDB0035858 & $\uparrow$ & $\downarrow^{* * *}$ \\
\hline 476.3109 & 9.42 & $\mathrm{C}_{21} \mathrm{H}_{41} \mathrm{~N}_{5} \mathrm{O}_{7}$ & Netilmicin & $+\mathrm{H}$ & HMDB0015090 & $\downarrow^{* * *}$ & $\downarrow^{* * *}$ \\
\hline 506.3557 & 11.46 & $\mathrm{C}_{26} \mathrm{H}_{52} \mathrm{NO}_{6} \mathrm{P}$ & LysoPC (P-18:1(9Z)) & $+\mathrm{H}$ & HMDB0010408 & $\downarrow^{*}$ & $\downarrow^{* *}$ \\
\hline 508.3734 & 11.45 & $\mathrm{C}_{26} \mathrm{H}_{54} \mathrm{NO}_{6} \mathrm{P}$ & LysoPC (P-18:0) & $+\mathrm{H}$ & HMDB0013122 & $\downarrow^{* * *}$ & $\downarrow^{* * *}$ \\
\hline 522.3512 & 10.58 & $\mathrm{C}_{26} \mathrm{H}_{52} \mathrm{NO}_{7} \mathrm{P}$ & LysoPC (18:1(11Z)) & $+\mathrm{H}$ & HMDB0010385 & $\downarrow$ & $\downarrow^{* * *}$ \\
\hline 546.3563 & 10.41 & $\mathrm{C}_{28} \mathrm{H}_{52} \mathrm{NO}_{7} \mathrm{P}$ & LysoPC $(20: 3(5 Z, 8 Z, 11 Z))$ & $+\mathrm{H}$ & HMDB0010393 & $\downarrow$ & $\downarrow^{* *}$ \\
\hline 548.3688 & 11.05 & $\mathrm{C}_{28} \mathrm{H}_{54} \mathrm{NO}_{7} \mathrm{P}$ & LysoPC $(20: 2(11 Z, 14 Z))$ & $+\mathrm{H}$ & HMDB0010392 & $\downarrow^{* *}$ & $\downarrow^{* * *}$ \\
\hline 550.3856 & 13.13 & $\mathrm{C}_{28} \mathrm{H}_{56} \mathrm{NO}_{7} \mathrm{P}$ & LysoPC (20:1(11Z)) & $+\mathrm{H}$ & HMDB0010391 & $\downarrow^{* * *}$ & $\uparrow^{*}$ \\
\hline 552.3995 & 15.09 & $\mathrm{C}_{28} \mathrm{H}_{58} \mathrm{NO}_{7} \mathrm{P}$ & LysoPC (20:0/0:0) & $+\mathrm{H}$ & HMDB0010390 & $\downarrow^{* * *}$ & $\downarrow^{* *}$ \\
\hline 570.3534 & 11.46 & $\mathrm{C}_{30} \mathrm{H}_{52} \mathrm{NO}_{7} \mathrm{P}$ & LysoPC $(22: 5(4 Z, 7 Z, 10 Z, 13 Z, 16 Z))$ & $+\mathrm{H}$ & HMDB0010402 & $\downarrow^{* *}$ & $\downarrow^{* * *}$ \\
\hline 576.3993 & 13.8 & $\mathrm{C}_{30} \mathrm{H}_{58} \mathrm{NO}_{7} \mathrm{P}$ & LysoPC $(22: 2(13 Z, 16 Z))$ & $+\mathrm{H}$ & HMDB0010400 & $\downarrow^{* *}$ & $\downarrow^{* *}$ \\
\hline 578.4157 & 15.18 & $\mathrm{C}_{30} \mathrm{H}_{60} \mathrm{NO}_{7} \mathrm{P}$ & LysoPC $(22: 1(13 Z))$ & $+\mathrm{H}$ & HMDB0010399 & $\downarrow^{* * *}$ & $\downarrow^{* *}$ \\
\hline 103.0412 & 1.35 & $\mathrm{C}_{4} \mathrm{H}_{8} \mathrm{O}_{3}$ & 2-Hydroxybutyric acid & $-\mathrm{H}$ & HMDB0000008 & $\downarrow^{* * *}$ & $\downarrow$ \\
\hline 279.2324 & 15.25 & $\mathrm{C}_{18} \mathrm{H}_{34} \mathrm{O}_{3}$ & Ricinoleic acid & $-\mathrm{H}_{2} \mathrm{O}-\mathrm{H}$ & HMDB0034297 & $\downarrow^{* * *}$ & $\uparrow$ \\
\hline 303.2339 & 14.95 & $\mathrm{C}_{20} \mathrm{H}_{32} \mathrm{O}_{2}$ & Arachidonic acid & $-\mathrm{H}$ & HMDB0001043 & $\downarrow^{* * *}$ & $\uparrow^{*}$ \\
\hline 347.221 & 15.26 & $\mathrm{C}_{21} \mathrm{H}_{34} \mathrm{O}_{5}$ & 5a-Tetrahydrocortisol & $-\mathrm{H}_{2} \mathrm{O}-\mathrm{H}$ & HMDB0000526 & $\downarrow^{* * *}$ & $\uparrow$ \\
\hline 353.1403 & 14.55 & $\mathrm{C}_{21} \mathrm{H}_{22} \mathrm{O}_{5}$ & Lansiumarin $\mathrm{C}$ & $-\mathrm{H}$ & HMDB0034838 & $\downarrow^{* * *}$ & $\uparrow$ \\
\hline 355.1575 & 16.57 & $\mathrm{C}_{21} \mathrm{H}_{24} \mathrm{O}_{5}$ & Chalepin acetate & $-\mathrm{H}$ & HMDB0030666 & $\downarrow^{* * *}$ & $\uparrow$ \\
\hline 371.2212 & 14.95 & $\mathrm{C}_{23} \mathrm{H}_{32} \mathrm{O}_{4}$ & Scutigeral & $-\mathrm{H}$ & HMDB0030012 & $\downarrow^{* * *}$ & $\uparrow$ \\
\hline 379.1573 & 15.25 & $\mathrm{C}_{20} \mathrm{H}_{28} \mathrm{O}_{5} \mathrm{~S}$ & Ecabet & $-\mathrm{H}$ & HMDB0015613 & $\downarrow^{* * *}$ & $\uparrow$ \\
\hline 403.1574 & 14.94 & $\mathrm{C}_{25} \mathrm{H}_{24} \mathrm{O}_{5}$ & Mammeigin & $-\mathrm{H}$ & HMDB0030785 & $\downarrow^{* * *}$ & $\uparrow$ \\
\hline 436.2818 & 10.94 & $\mathrm{C}_{21} \mathrm{H}_{44} \mathrm{NO}_{6} \mathrm{P}$ & PE (P-16:0e/0:0) & $-\mathrm{H}$ & HMDB0011152 & $\downarrow^{*}$ & $\uparrow^{* * *}$ \\
\hline 480.3112 & 10.16 & $\mathrm{C}_{23} \mathrm{H}_{48} \mathrm{NO}_{7} \mathrm{P}$ & LysoPE (18:0/0:0) & $-\mathrm{H}$ & HMDB0011130 & $\downarrow^{* * *}$ & $\uparrow^{* * *}$ \\
\hline 506.3291 & 10.95 & $\mathrm{C}_{25} \mathrm{H}_{50} \mathrm{NO}_{7} \mathrm{P}$ & LysoPE (0:0/20:1(11Z)) & $-\mathrm{H}$ & HMDB0011482 & $\downarrow^{* * *}$ & $\uparrow^{* * *}$ \\
\hline 528.3121 & 9.82 & $\mathrm{C}_{27} \mathrm{H}_{48} \mathrm{NO}_{7} \mathrm{P}$ & LysoPE $(0: 0 / 22: 4(7 Z, 10 Z, 13 Z, 16 Z))$ & $-\mathrm{H}$ & HMDB0011493 & $\downarrow^{* *}$ & $\uparrow^{* * *}$ \\
\hline
\end{tabular}

\section{Discussion}

To clarify the effects of GEB on the treatment of cerebral ischemia-reperfusion injury, we performed pharmacodynamic experiments and detected biochemical and histopathological indicators. Our results suggested that cerebral ischemia and reperfusion injury cause local oxidative stress and inflammatory injury, leading to apoptosis, autophagy, and necrosis. After cerebral ischemia, oxidants are overproduced because the redox state in the natural endogenous antioxidant system is in disequilibrium, and some studies have shown that the phenolic components of Gastrodia elata stimulate endogenous antioxidative responses, thereby attenuating neuronal damage. ${ }^{34,35}$ SOD, GSH-Px and other endogenous antioxidant enzymes play an important role in oxidant and redox balance. ${ }^{36}$ The overexpression of SOD after cerebral ischemic injury can reduce mitochondrial dysfunction and apoptosis. A large number of studies have shown that ischemic injury induces polyunsaturated fatty acid lipid peroxidation to produce high levels of $\mathrm{MDA}^{37}$ leading to decreased membrane mobility and permeability. The levels of MDA are thus closely related to the degree of cell injury and oxidative stress. Oxidative stress is closely related to the injury caused by the excessive secretion of inflammatory cytokines, TNF- $\alpha$. As a major cause of cerebral infarction, the secretion and synthesis of TNF- $\alpha$ increase ${ }^{38,39}$ the permeability of vascular endothelial cells, enhance the aggregation and activation of leukocytes, and increase inflammatory mediator release. These cytokines induce the expression of chemokines and cell adhesion molecules, which leads to leukocyte infiltration, leukocyte aggregation and the release of inflammatory mediators leading to blood-brain barrier leakage. IL-10 strongly inhibits the oversecretion of TNF- $\alpha$ and IL-1 $\beta$ at the transcriptional level and plays an anti-inflammatory role. Anti-oxidation and anti- 


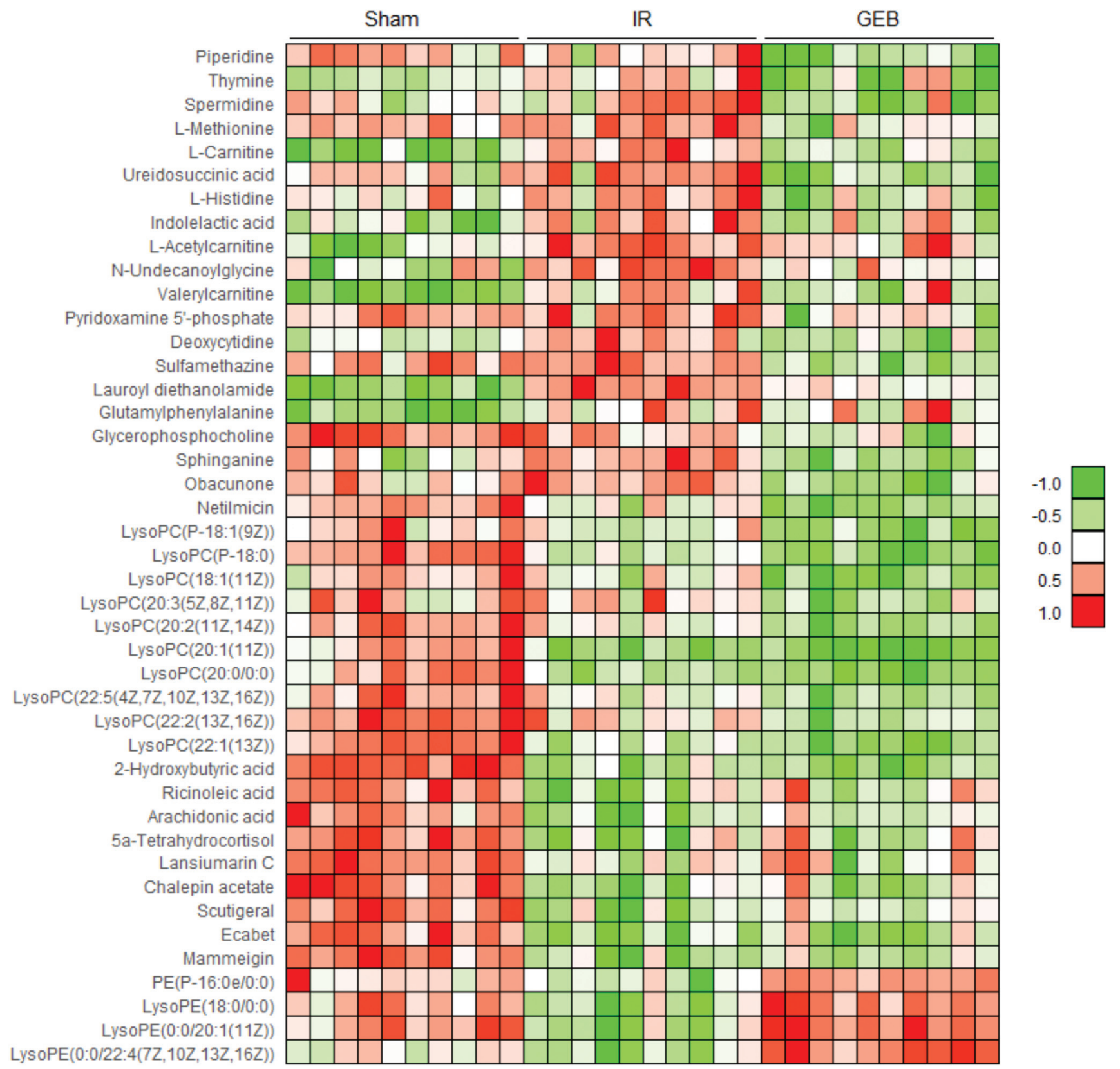

Fig. 7 Heat map of metabolites in the cerebral ischemic injury of rats from sham, IR and GEB groups. Data (peak areas) were normalized between -1 and 1 (green: the lowest level and red: the highest level).

inflammation are the proposed mechanisms of GEB activity during ischemia-reperfusion injury.

Changes in the metabolic environment of the cerebrospinal fluid after cerebral ischemia have been reported. Studies on the metabolomic response to GEB during the treatment of ischemic disease are relatively sparse. In this study, six metabolites and their associated metabolic pathways were identified via UPLC-Q-TOF/MS-based metabolomic analysis.

This study verified the effect of Gastrodia elata on the treatment of cerebral ischemia-reperfusion injury based on metabolomics. However, there were uncertainties in the singlegroup analysis. Multi-omics integrative analysis methods can improve the effectiveness of screening and enhance the accuracy of disease predictions; in future studies, transcriptomics will be performed to complement our datasets to reveal the mechanism(s) of disease development at the genetic level.

Studies have shown that a large number of free radicals are produced during cerebral ischemia, leading to neurotoxicity by acting on polyunsaturated fatty acids to cause lipid peroxidation or inducing the cross-linking of macromolecules (including DNA, RNA and amino acids). Arachidonic acid (ARA) is an omega-6 polyunsaturated fatty acid that is produced from linoleic acid; ${ }^{40,41}$ the levels of ARA in the IR group were significantly lower than those in the sham group 


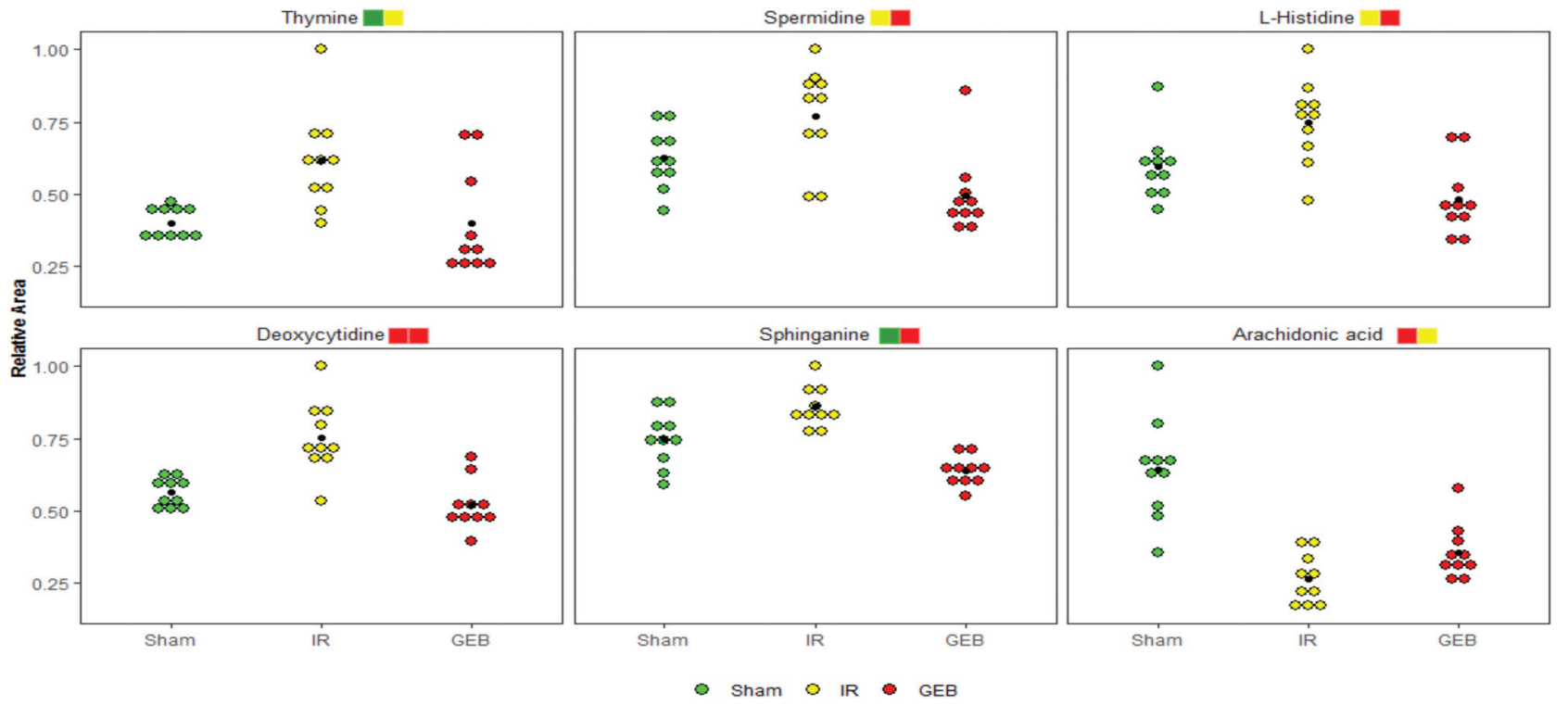

Fig. 8 Relative levels of the selected 6 metabolites in the plasma of rats from sham, IR and GEB groups. Significant levels ( $t$-test): ${ }^{*} P<0.05,{ }^{* *} p<0.01$, ${ }^{* * *} p<0.001$ are represented by yellow, green and red squares, respectively, and the default front and back order is IR vs. sham and GEB vs. IR, respectively.

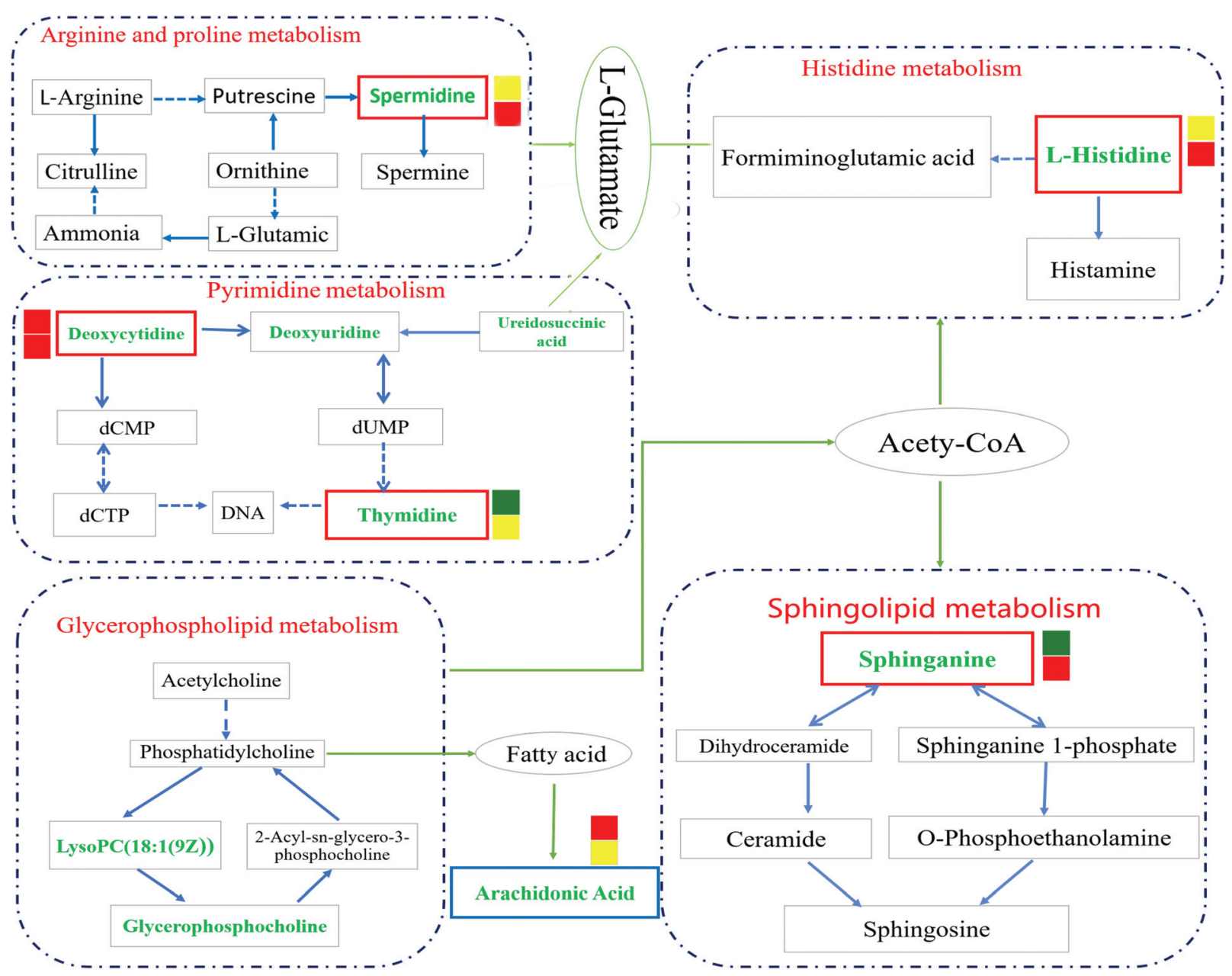

Fig. 9 Overview of metabolic pathway analysis. The green font was identified as the metabolite; after the intervention of Gastrodia elata, the level of metabolites in the red border decreased and that of the blue border increased. Significance: ${ }^{*} P<0.05,{ }^{* *} p<0.01,{ }^{\star \star *} p<0.001$ are represented by yellow, green and red squares, respectively, and the upper and lower default is IR vs. sham and GEB vs. IR, respectively. 
$(p<0.001)$, whilst the content of ARA in the GEB group was significantly higher than that in the IR group $(p<0.05)$. This suggests that GEB regulates arachidonic acid metabolism by reducing lipid peroxidation induced by free radicals to protect against cerebral ischemia-reperfusion injury.

Histamine release is known to increase following cerebral ischemia-reperfusion injury in rats, which is consistent with the significant increase in L-histidine observed in this study $(p<0.05)$. The increase in histidine leads to the increase of histamine formation which may promote further inflammation. $^{42,43}$ Following GEB treatment, the levels of L-histidine significantly decreased $(p<0.001)$ suggesting that GEB regulates histidine metabolism.

Sphingolipid metabolism affects neuronal function by regulating synaptic activity and APP processing. ${ }^{44}$ Sphinganine (SG) is a major component of ceramide that induces the production of inflammatory factors related to neuroinflammation. Studies have demonstrated ${ }^{45}$ that increased levels of ceramide induce neuronal apoptosis. In addition, SG influences metabolism through the inhibition of esterification and cholesterol transport. As a component of membranes and lipoproteins, SG regulates cell proliferation, differentiation, adhesion, migration, membrane transport, transcriptional activity, autophagy and apoptosis, all of which are relevant to autoimmune and inflammatory disease. ${ }^{46,47}$ The levels of SG increased after cerebral ischemia-reperfusion $(P<0.01)$, which may be related to the neurotoxicity and neuroinflammation caused by elevated ceramide levels. The dramatic decrease in SG levels $(P<$ 0.001) following GEB intervention through regulation of the sphingomyelin metabolic pathway is thus likely to contribute to its therapeutic effects.

Deoxycytidine (DD) is a major nucleoside of deoxyribonucleic acid (DNA), which is composed of cytosine and deoxyribose. Thymine is a pyrimidine base which differs from uracil as the fifth hydrogen is replaced by a methyl group. As a human metabolite, DD is present in all organisms and natural compounds. Exogenous uridine and cytidine are necessary for the maintenance of brain function in rats with ischemia-reperfusion injury. In this study, the levels of plasma DD and thymine in the IR group were significantly higher than those of the sham group $(P<0.001 ; P<0.01)$, and a significant correction after GEB treatment was observed $(P<0.001 ; P<0.05)$. This suggests that the mechanism of GEB in the treatment of cerebral ischemia-reperfusion injury may be related to the regulation of DD and thymine levels through the pyrimidine metabolic pathway.

Spermidine (SD) is a natural polyamine that is widely distributed in mammalian cells. SD prolongs the lifespan of model organisms by inducing autophagy and preventing memory loss during aging. We found that the spermidine content in the IR group was higher than that of the sham group $(p<0.05)$, which was consistent with previous studies. This may be because in cases of cerebral ischemia, SD is released from neurons to the extracellular chamber and cleared into the bloodstream. ${ }^{48}$ The content of SD in the GEB group was significantly lower than that of the IR group $(p<0.001)$.

\section{Conclusions}

In summary, we used metabolomics with LC-MS technology to investigate the mechanisms of GEB on cerebral ischemiareperfusion injury in rats. Pharmacodynamics and metabolomics confirmed that GEB could improve cerebral ischemia through the regulation of metabolism in vivo. Consistent with previous studies, the mechanisms of GEB in the treatment of cerebral ischemia were related to reduced inflammation, oxidation, neurotoxicity, and apoptosis.

Metabolomics analysis showed that GEB could improve cerebral ischemia-reperfusion injury in rats through its regulation of arachidonic acid metabolism, histidine metabolism, pyrimidine metabolism, arginine and proline metabolism, sphingolipid metabolism, and glycerophospholipid metabolism. We also observed differences in the levels of arachidonic acid, L-histidine, sphinganine, thymine, spermidine, and deoxycytidine before and after GEB treatment. These data highlight these metabolites as potential biomarkers of GEB treatment, which provides a reference for the treatment of ischemic disease.

\section{Conflicts of interest}

There are no conflicts to declare.

\section{Acknowledgements}

Financial support by the National Natural Science Foundation of China (No. 81560638), Young Scientists Training Project of Jiangxi Province (No. 20162BCB23003), Jiangxi Province 5511 R\&D Projects (No. 20165BCB19009), Jiangxi Province Talent Project (GanRenSheZi No. [2016]332), and Nanchang Innovative Talent Team (No. [2018]274) is acknowledged.

\section{References}

1 S. Fu, L. Chen, Y. Wu, Y. Tang, L. Tang, Y. Zhong, S. Wang, H. Liu, X. Wang and A. Chen, Gastrodin pretreatment alleviates myocardial ischemia/reperfusion injury through promoting autophagic flux, Biochem. Biophys. Res. Commun., 2018, 2421-2428, DOI: 10.1016/j.bbrc.2018.06.171.

2 R. Maheswaran, T. Pearson, M. J. Campbell, R. P. Haining, C. W. McLeod, N. Smeeton and C. D. Wolfe, A protocol for investigation of the effects of outdoor air pollution on stroke incidence, phenotypes and survival using the South London Stroke Register, Int. J. Health Geogr., 2006, 5-10, DOI: $10.1186 / 1476-072 X-5-10$.

3 C. L. Allen and U. Bayraktutan, Risk factors for ischaemic stroke, Int. J. Stroke, 2008, 105-116, DOI: 10.1111/j.17474949.2008.00187.x.

4 S. N. Chapman, P. Mehndiratta, M. C. Johansen, T. L. McMurry, K. C. Johnston and A. M. Southerland, Current perspectives on the use of intravenous recombi- 
nant tissue plasminogen activator (tPA) for treatment of acute ischemic stroke, Vasc. Health Risk Manage., 2014, 7587, DOI: 10.2147/VHRM.S39213.

5 H. Chen, B. Guan, X. Chen, X. Chen, C. Li, J. Qiu, D. Yang, K. J. Liu, S. Qi and J. Shen, Baicalin Attenuates Blood-Brain Barrier Disruption and Hemorrhagic Transformation and Improves Neurological Outcome in Ischemic Stroke Rats with Delayed t-PA Treatment: Involvement of ONOO (-)-MMP-9 Pathway, Transl. Stroke Res., 2018, 515-529, DOI: 10.1007/s12975-017-0598-3.

6 X. Zeng, S. Zhang, L. Zhang, K. Zhang and X. Zheng, A study of the neuroprotective effect of the phenolic glucoside gastrodin during cerebral ischemia in vivo and in vitro, Planta Med., 2006, 1359-1365, DOI: 10.1055/ s-2006-951709.

7 X. Zheng and J. Liu, The Technology Research for Gastrodia elata.BL Health Protection Beverage Production, Food Sci., 2005, 653-654.

8 F. He, X. Duan, R. Dai, W. Wang, C. Yang and Q. Lin, Protective Effects of Ethyl Acetate Extraction from Gastrodia Elata Blume on Blood-Brain Barrier in Focal Cerebral Ischemia Reperfusion, Afr. J. Tradit., Complementary Altern. Med., 2016, 199-209, DOI: 10.21010/ ajtcam.v13i4.26.

9 S. M. Huang, F. Xu, S. H. Lam, Z. Gong and C. N. Ong, Metabolomics of developing zebrafish embryos using gas chromatography- and liquid chromatography-mass spectrometry, Mol. BioSyst., 2013, 1372-1380, DOI: 10.1039/ c3mb25450j.

10 B. Liu, F. Li, J. Shi, D. Yang, Y. Deng and Q. Gong, Gastrodin ameliorates subacute phase cerebral ischemia reperfusion injury by inhibiting inflammation and apoptosis in rats, Mol. Med. Rep., 2016, 4144-4152, DOI: 10.3892/ mmr.2016.5785.

11 X. S. Wang, Z. Tian, N. Zhang, J. Han, H. L. Guo, M. G. Zhao and S. B. Liu, Protective Effects of Gastrodin Against Autophagy-Mediated Astrocyte Death, Phytother. Res., 2016, 386-396, DOI: 10.1002/ptr.5538.

12 S. K. McCann and C. L. Roulston, NADPH Oxidase as a Therapeutic Target for Neuroprotection against Ischaemic Stroke: Future Perspectives, Brain Sci., 2013, 561-598, DOI: 10.3390/brainsci3020561.

13 L. Luo, L. Zhen, Y. Xu, Y. Yang, S. Feng, S. Wang and S. Liang, (1)H NMR-based metabonomics revealed protective effect of Naodesheng bioactive extract on ischemic stroke rats, J. Ethnopharmacol., 2016, 257-269, DOI: 10.1016/j.jep.2016.03.059.

14 M. A. Moskowitz, E. H. Lo and C. Iadecola, The science of stroke: mechanisms in search of treatments, Neuron, 2010, 181-198, DOI: 10.1016/j.neuron.2010.07.002.

15 H. Chen, H. Yoshioka, G. S. Kim, J. E. Jung, N. Okami, H. Sakata, C. M. Maier, P. Narasimhan, C. E. Goeders and P. H. Chan, Oxidative Stress in Ischemic Brain Damage: Mechanisms of Cell Death and Potential Molecular Targets for Neuroprotection, Antioxid. Redox Signaling, 2010, 14, 1505-1517.
16 F. C. Barone and G. Z. Feuerstein, Inflammatory Mediators and Stroke: New Opportunities for Novel Therapeutics, J. Cereb. Blood Flow Metab., 1999, 819-834.

17 A. S. Go, D. Mozaffarian and V. L. Roger, Executive summary: heart disease and stroke statistics-2013 update: a report from the American Heart Association, Circulation, 2013, 127, 143-152.

18 P. Ramana, E. Adams, P. Augustijns and A. Van Schepdael, Metabonomics and drug development, Methods Mol. Biol., 2015, 1277, 195-207.

19 D. Feng, B. Wang, L. Wang, N. Abraham, K. Tao, L. Huang, W. Shi, Y. Dong and Y. Qu, Pre-ischemia melatonin treatment alleviated acute neuronal injury after ischemic stroke by inhibiting endoplasmic reticulum stress-dependent autophagy via PERK and IRE1 signalings, J. Pineal Res., 2017, 62(3), e12395.

20 C. Tang, L. Wang, J. Li, X. Liu, M. Cheng and H. Xiao, Analysis of the metabolic profile of parishin by ultra-performance liquid chromatography/quadrupole-time of flight mass spectrometry, Biomed. Chromatogr., 2015, 29, 19131920.

21 M. Jiang, Q. Wang, J. Chen, Y. Wang, G. Fan and Y. Zhu, Comparative metabonomics of Wenxin Keli and Verapamil reveals differential roles of gluconeogenesis and fatty acid beta-oxidation in myocardial injury protection, Sci. Rep., 2017, 7, 8739.

22 Q. Guan, S. Liang, Z. Wang, Y. Yang and S. Wang, (1)H NMR-based metabonomic analysis of the effect of optimized rhubarb aglycone on the plasma and urine metabolic fingerprints of focal cerebral ischemia-reperfusion rats, J. Ethnopharmacol., 2014, 154, 65-75.

23 D. W. Howells, M. J. Porritt, S. S. Rewell, V. O'Collins, E. S. Sena, H. B. van der Worp, R. J. Traystman and M. R. Macleod, Different strokes for different folks: the rich diversity of animal models of focal cerebral ischemia, J. Cereb. Blood Flow Metab., 2010, 1412-1431, DOI: 10.1038/ jcbfm.2010.66.

24 G. M. Xi, H. Q. Wang, G. H. He, C. F. Huang and G. Y. Wei, Evaluation of murine models of permanent focal cerebral ischemia, Chin. Med. J., 2004, 117, 389-394.

25 O. U. Scremin and D. J. Jenden, Focal ischemia enhances choline output and decreases acetylcholine output from rat cerebral cortex, Stroke, 1989, 20, 92-95.

26 X. Zhang, M. He, S. Lei, B. Wu, T. Tan, H. Ouyang, W. Xu and Y. Feng, An integrative investigation of the therapeutic mechanism of Ainsliaea fragrans Champ. in cervicitis using liquid chromatography tandem mass spectrometry based on a rat plasma metabolomics strategy, J. Pharm. Biomed. Anal., 2018, 221-231, DOI: 10.1016/j.jpba.2018.04.048.

27 H. G. Gika, G. A. Theodoridis, J. E. Wingate and I. D. Wilson, Within-day reproducibility of an HPLC-MSbased method for metabonomic analysis: application to human urine, J. Proteome Res., 2007, 6, 3291-3303.

28 A. Zhang, H. Sun and X. Wang, Mass spectrometry-driven drug discovery for development of herbal medicine, Mass Spectrom. Rev., 2018, 307-320, DOI: 10.1002/mas.21529. 
29 G. A. Theodoridis, H. G. Gika, E. J. Want and I. D. Wilson, Liquid chromatography-mass spectrometry based global metabolite profiling: a review, Anal. Chim. Acta, 2012, 711, 7-16.

30 M. Kanehisa and S. Goto, KEGG: Kyoto Encyclopaedia of Genes and Genomes, Nucleic Acids Res., 2000, 28, 27-30.

$31 \mathrm{~J}$. Chong and J. Xia, MetaboAnalystR: an R package for flexible and reproducible analysis of metabolomics data, Bioinformatics, 2018, 4313-4314, DOI: 10.1093/bioinformatics/bty528.

32 T. Jewison, Y. Su, F. M. Disfany, Y. Liang, C. Knox, A. Maciejewski, J. Poelzer, J. Huynh, Y. Zhou, D. Arndt, Y. Djoumbou, Y. Liu, L. Deng, A. C. Guo, B. Han, A. Pon, M. Wilson, S. Rafatnia, P. Liu and D. S. Wishart, SMPDB 2.0: big improvements to the Small Molecule Pathway Database, Nucleic Acids Res., 2013, D478-D484, DOI: 10.1093/nar/gkt1067.

33 S. Bonny, L. Paquin, D. Carrie, J. Boustie and S. Tomasi, Ionic liquids based microwave-assisted extraction of lichen compounds with quantitative spectrophotodensitometry analysis, Anal. Chim. Acta, 2011, 707, 69-75.

34 A. H. Shi, J. M. Xiang, F. Y. He, Y. P. Zhu, G. B. Zhu, Y. H. Lin and N. N. Zhou, The Phenolic Components of Gastrodia elata improve Prognosis in Rats after Cerebral Ischemia/Reperfusion by Enhancing the Endogenous Antioxidant Mechanisms, Oxid. Med. Cell. Longevity, 2018, 7642158, DOI: 10.1155/2018/7642158.

35 F. He, X. Duan, R. Dai, Y. Li and Q. Lin, Protective Effect of 4-Methoxy Benzyl Alcohol on the Blood-Brain Barrier after Cerebral Ischemia Reperfusion Injury, J. Stroke Cerebrovasc. Dis., 2017, 26, 1258-1265.

36 A. Djordjevic, S. Spasic, A. Jovanovic-Galovic, R. Djordjevic and G. Grubor-Lajsic, Oxidative stress in diabetic pregnancy: SOD, CAT and GSH-Px activity and lipid peroxidation products, J. Matern.-Fetal Neonat. Med., 2004, 367372, DOI: 10.1080/14767050400018270.

37 H. Chen, Y. S. Song and P. H. Chan, Inhibition of NADPH oxidase is neuroprotective after ischemia-reperfusion, J. Cereb. Blood Flow Metab., 2009, 1262-1272, DOI: 10.1038/ jcbfm.2009.47.

38 M. Castellanos, C. José and G. María, InflammationMediated Damage in Progressing Lacunar Infarctions A Potential Therapeutic Target, Stroke, 2002, 982-987.
39 T. Sairanen, O. Carpen and M. Karjalainenlindsberg, Evolution of cerebral tumor necrosis factor-XMLLink_XYZ production during human ischemic stroke, Stroke, 2001, 1750-1758.

40 C. Wang, C. Liu, M. Wang, Q. Ma, Y. Li, T. Wang and B. Zhao, UPLC-HRMS-Based Plasma Metabolomic Profiling of Novel Biomarkers by Treatment with KDZI in Cerebral Ischemia Reperfusion Rats, Molecules, 2018, 1315, DOI: 10.3390/molecules23061315.

41 M. N. Hoda, I. Singh, A. K. Singh and M. Khan, Reduction of lipoxidative load by secretory phospholipase A2 inhibition protects against neurovascular injury following experimental stroke in rat, J. Neuroinflammation, 2009, 6, 21.

42 N. Hirasawa, Expression of Histidine Decarboxylase and Its Roles in Inflammation, Int. J. Mol. Sci., 2019, 376388.

43 L. M. Kager, D. C. Blok, I. O. Lede, W. Rahman, R. Afroz, P. Bresser, J. S. van der Zee, A. Ghose, C. E. Visser, M. D. de Jong, M. W. Tanck, A. S. Zahed, K. M. Alam, M. Hassan, A. Hossain, R. Lutter, C. V. Veer, A. M. Dondorp, J. C. Meijers and T. van der Poll, Pulmonary tuberculosis induces a systemic hypercoagulable state, J. Infect., 2015, 324-334, DOI: 10.1016/j.jinf.2014.10.006.

44 N. J. Haughey, V. V. Bandaru, M. Bae and M. P. Mattson, Roles for dysfunctional sphingolipid metabolism in Alzheimer's disease neuropathogenesis, Biochim. Biophys. Acta, 2010, 1801, 878-886.

45 W. Paschen, L. Csiba, G. Röhn and D. Bereczki, Polyamine metabolism in transient focal ischemia of rat brain, Brain Res., 1991, 566, 354-355.

46 L. Davies, K. Fassbender and S. Walter, Sphingolipids in Neuroinflammation, in Sphingolipids in Disease, Springer, Vienna, 2013, pp. 421-430.

47 A. H. Merrill Jr., T. H. Stokes, A. Momin, H. Park, B. J. Portz, S. Kelly, E. Wang, M. C. Sullards and M. D. Wang, Sphingolipidomics: a valuable tool for understanding the roles of sphingolipids in biology and disease, J. Lipid Res., 2009, S97-102, DOI: 10.1194/jlr.R800073-JLR200.

48 L. R. Ballou, S. J. F. Laulederkind, E. F. Rosloniec and R. Raghow, Ceramide signalling and the immune response, Biochim. Biophys. Acta, Lipids Lipid Metab., 1996, 1301, 273287. 